\title{
Bilateral uniportal video-assisted thoracic surgery segmentectomy S3: an unusual case
}

\author{
Alonso José Oviedo Argueta ${ }^{1,2}$, Sonia Raquelline Roque Cañas ${ }^{1,2}$, Yuming Zhu' ${ }^{2}$, Diego Gonzalez-Rivas ${ }^{2,3,4}$ \\ ${ }^{1}$ Department of Surgery, Salvadoran Social Security Institute (ISSS), San Salvador, El Salvador; ${ }^{2}$ Department of Thoracic Surgery, Shanghai \\ Pulmonary Hospital of Tongji University, Shanghai 200000, China; ${ }^{3}$ Department of Thoracic Surgery Coruña University Hospital, Coruña, Spain; \\ ${ }^{4}$ Department of Thoracic Surgery and Minimally Invasive Thoracic Surgery Unit (UCTMI), Coruña, Spain \\ Correspondence to: Diego Gonzalez-Rivas. Department of Thoracic Surgery Coruña University Hospital, Coruña, Spain. Email: diego.gonzalez.rivas@sergas.es.
}

\begin{abstract}
When talking about lung cancer, it is important to recognize this as the first cause of death of neoplastic origin. The detection of this in early stages has made the emergence of ground glass opacity (GGO) more frequent due to the establishment of lung cancer screening programs, allowing the reduction of morbidity and mortality caused by the same and achieving a curative treatment of it. The management of multiple GGOs depends much on the characteristics of these, however, being multiple and contralateral should be considered surgical resection, always taking into account the stage of the dominant lesion. In this article, we present a case of a 60 years old woman with a bilateral GGO lesions located in segment 3 on both sides. A bilateral uniportal video-assisted thoracic surgery (VATS) anatomic segmentectomy S3 of both lesions was performed in a single stage surgery. The postoperative course of the patient was uneventful.
\end{abstract}

Keywords: Segmentectomy; sublobar resection; bilateral; uniportal video-assisted thoracic surgery (VATS); multiple; ground glass opacity (GGO)

Received: 15 October 2017; Accepted: 18 October 2017; Published: 11 November 2017.

doi: 10.21037 /jovs.2017.10.13

View this article at: http://dx.doi.org/10.21037/jovs.2017.10.13

\section{Introduction}

The presence of ground glass opacity (GGO) in a chest computed tomography scan (CT Scan) is a radiological entity that has been booming, mostly after the initiation of screening tests for lung cancer (1-3). Particularly the GGO lesions have been related with non-small cell lung cancers (NSCLCs) specifically the lepidic component of adenocarcinomas $(1,2)$. It is necessary to classify this according to its radiological characteristics. The pathological-radiological correlation allows to make an adequate treatment $(1,4,5)$, observing that the presence of the GGO component is a prognostic factor of cancer in early stages (6-8). Likewise, the appropriate follow-up in combination with the resections of this type of lesions actually is a challenge for thoracic surgeons, even more in cases of persistent or multiples GGOs (9). however, nowadays we observe that sublobar resections [single or combined segments (10)] are rising and giving adequate oncological results and in terms of survival (9,11-13). We present a case of a patient with unusual multiple GGOs placed in the anterior segment in both sides.

\section{Patient and workup}

A 50 years old female patient, without previous medical history or symptoms, which screening CT scan shows multiple GGOs in both sides with different diameters, in the right side one of $10 \mathrm{~mm}$, and in the left side two of 14 and $5 \mathrm{~mm}$ respectively (Figure 1). She was followed for 3 months with persistence of these lesions. The bronchoscopy and positron emission tomography (PET) CT Scan were not realized. Forced expiratory volume in 1 second (FEV1) was $89.3 \%$. She was planned to perform both resections by a single stage bilateral uniportal video-assisted thoracic surgery (VATS) approach.

\section{Pre-operative preparation}

The operation was scheduled to perform bilateral anterior 

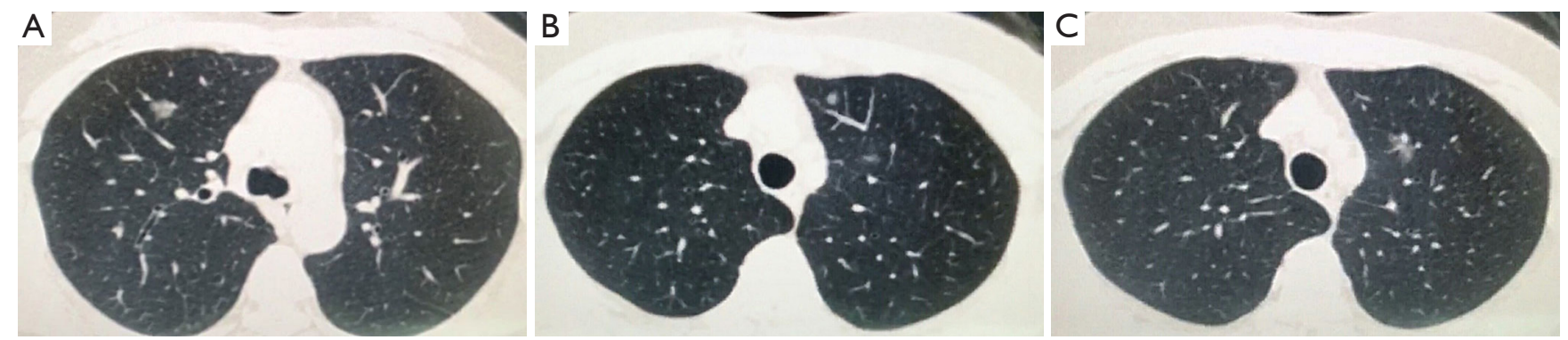

Figure 1 Preoperative CT scan shows multiple GGOs in both sides. (A) GGO in the right anterior segment (S3): $10 \mathrm{~mm}$ of diameter; (B) GGO in the left anterior segment (S3): $5 \mathrm{~mm}$ of diameter; (C) GGO in the left anterior segment (S3) dominant lesion: $14 \mathrm{~mm}$ of diameter. GGO, ground glass opacity.
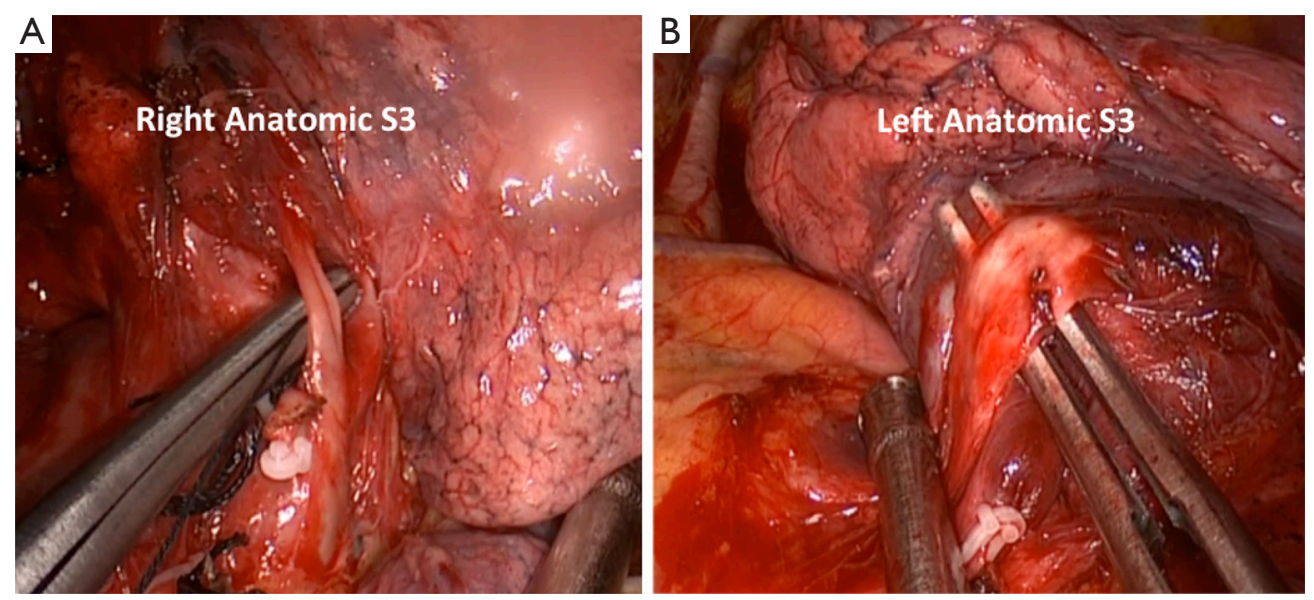

Figure 2 Surgical image of right S3 during vessel dissection (A) and left S3 during bronchus dissection (B).

segmentectomies via uniportal VATS. The patient was intubated with a double lumen endotracheal tube. Prophylaxis with cephalosporin, central venous pressure line and urinary catheters were inserted before the surgery.

\section{Equipment performance card}

Full HD Thoracoscopic camera (Karl Stoz ${ }^{\circledR}$ ), Uniportal VATS Instruments (Scanlan ${ }^{\circledR}$ ), Wound Protector (Changzhou Company LTd.), Endo GIA ${ }^{\mathrm{TM}}$ staplers (Covidien-Medtronic), Advanced Energy Device HARMONIC ${ }^{\circledR}$ (Ethicon).

\section{Procedure}

Under general anesthesia and left lateral decubitus position, a right uniportal VATS approach through a $3-\mathrm{cm}$ incision was performed. Firstly, we dissected the right upper lobe vein and anterior arterial trunk. We proceeded to open the minor fissure, identification of the vessels of $\mathrm{S} 3$; $\mathrm{V} 3$ and A3 both with two branches (V3a, V3b, A3a and A3b respectively) (Figure $2 A$ ), ligation with silk and vascular clips and division of B3 with endo-staplers. In this side, we completed the segmentectomy cutting the parenchyma with endo-staplers. A chest tube (18 french) was placed at the end of the procedure. The patient was turned to a right lateral decubitus position and a $3-\mathrm{cm}$ incision was performed on the left side, fifth intercostal space, middle axillary line. A left uniportal VATS anatomic segmentectomy S3 was performed. We started opening the pleura over the vessels in the left upper lobe. The we dissected and ligated V3 and then the B3 was dissected and divided with an endostapler (Figure 2B) This maneuver exposed the artery of the segment (A3) and was divided like in the right side. The inflation and deflation of the lung to demark the limits of the segment was the last step. It was finalized by cutting the lung parenchyma following this landmarks and leaving 


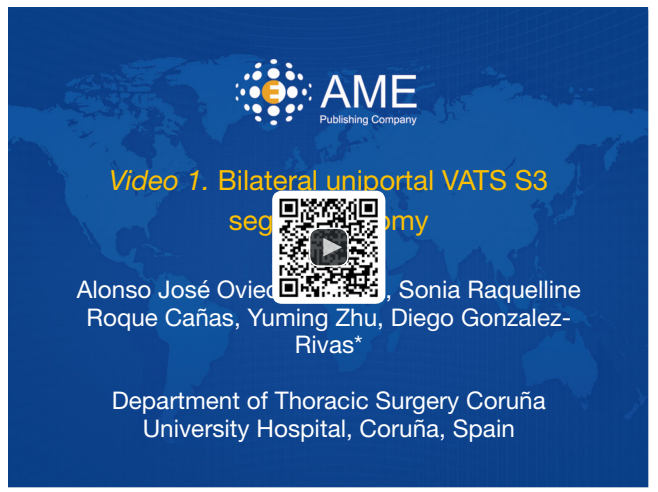

Figure 3 Bilateral uniportal VATS S3 segmentectomy (14). Available online: http://www.asvide.com/articles/1818

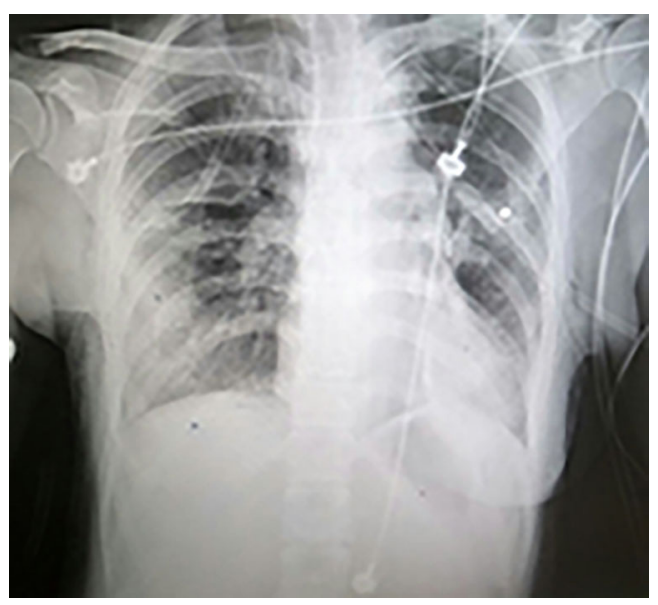

Figure 4 Post-operative X-chest ray

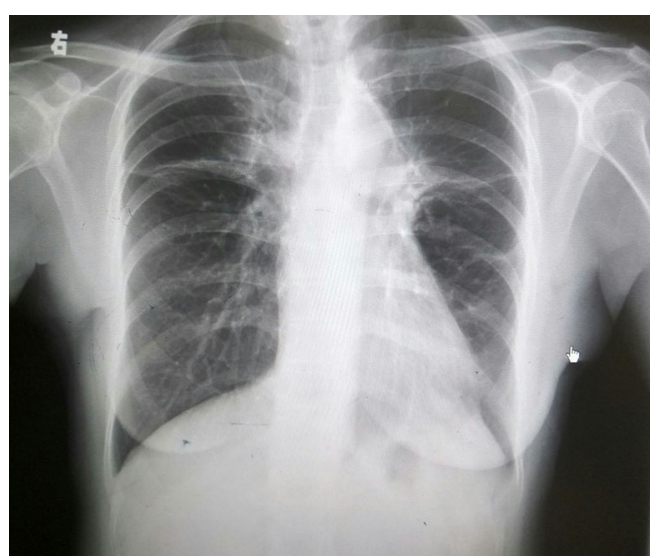

Figure $5 \mathrm{X}$-chest ray after removal of the chest drainages.

(C) Journal of Visualized Surgery. All rights reserved. the distal stump on the resected segment. Hemostasis was proved, and air leak excluded in both sides. We placed $18 \mathrm{~F}$ chest tube and the wound was closed. The estimated blood loss during the surgery was $50 \mathrm{~mL}$ and the total surgical time for both procedures was 90 minutes (Figure 3).

\section{Post-operative management}

The post-operative course of the patient was successful without complications. The management of the pain was with patients controlled analgesia (PCA). The post-operative $\mathrm{X}$-ray showed complete expanded lungs without complications (Figure 4). The chest tubes were removed in the $3 \mathrm{rd}$ day post-operatory (Figure 5), and the patient was discharged the same day without complications.

The paraffin biopsy results were in the right anterior segment: the bigger one was invasive adenocarcinoma, with $3 \mathrm{~cm}$ of margin. In the left anterior segment, the bigger was minimally invasive adenocarcinoma (MIA) and the other was adenocarcinoma in situ (AIS) with $2 \mathrm{~cm}$ of margin.

\section{Tips, tricks and pitfalls}

* A complete dissection of the distal part of the vessels provides a better and clear view before transection.

* Perform segmentectomies require the perfect knowledge of the distal bronchovascular anatomy of the lung.

* Taking into account the anatomical variant of the vessels is important to avoid mistakes.

* The uniportal VATS approach provides to the thoracic surgeon the flexibility to perform bilateral segmentectomies in a single stage surgical procedure.

\section{Acknowledgements}

None.

\section{Footnote}

Conflicts of Interest: The authors have no conflicts of interest to declare.

Informed Consent: Written informed consent was obtained from the patient for publication of this manuscript and any accompanying images. 


\section{References}

1. Suzuki, K. Whack-a-mole strategy for multifocal ground glass opacities of the lung. J Thorac Dis 2017;9:S201-7.

2. Kobayashi Y, Mitsudomi T. Management of ground-glass opacities: should all pulmonary lesions with ground-glass opacity be surgically resected? Transl Lung Cancer Res 2013;2:354-63.

3. Moon Y, Sung SW, Lee KY, et al. Clinicopathological characteristics and prognosis of non-lepidic invasive adenocarcinoma presenting as ground glass opacity nodule. J Thorac Dis 2016;8:2562-70.

4. Suzuki K, Kusumoto M, Watanabe S, et al. Radiologic classification of small adenocarcinoma of the lung: radiologic-pathologic correlation and its prognostic impact. Ann Thorac Surg 2006;81:413-9.

5. Liu LH, Liu M, Wei R, et al. CT findings of persistent pure ground glass opacity: can we predict the invasiveness?. Asian Pac J Cancer Prev 2015;16:1925-8.

6. Kim HY, Shim YM, Lee KS, et al. Persistent pulmonary nodular ground-glass opacity at thin-section CT: histopathologic comparisons. Radiology 2007;245:267-75.

7. Lee HY, Lee KS. Ground-glass opacity nodules: histopathology, imaging evaluation, and clinical

doi: 10.21037/jovs.2017.10.13

Cite this article as: Argueta AJ, Cañas SR, Zhu Y, GonzalezRivas D. Bilateral uniportal video-assisted thoracic surgery segmentectomy S3: an unusual case. J Vis Surg 2017;3:168. implications. J Thorac Imaging 2011;26:106-18.

8. Hattori A, Matsunaga T, Takamochi K, et al. Prognostic impact of a ground glass opacity component in the clinical T classification of non-small cell lung cancer. J Thorac Cardiovasc Surg 2017. [Epub ahead of print].

9. Hattori A, Matsunaga T, Takamochi K,et al. Surgical management of multifocal ground-glass opacities of the lung: correlation of clinicopathologic and radiologic findings. Thorac Cardiovasc Surg 2017;65:142-9.

10. González-Rivas D, Lirio F, Sesma J. Uniportal anatomic combined unusual segmentectomies. J Vis Surg 2017;3:91.

11. Tsutani Y, Miyata Y, Nakayama H, et al. Appropriate sublobar resection choice for ground glass opacitydominant clinical stage IA lung adenocarcinoma: wedge resection or segmentectomy. Chest 2014;145:66-71.

12. Cho JH, Choi YS, Kim J, et al. Long-term outcomes of wedge resection for pulmonary ground-glass opacity nodules. Ann Thorac Surg 2015;99:218-22.

13. Okada M, Mimae T, Tsutni Y, et al. Segmentectomy versus lobectomy for clinical stage IA lung adenocarcinoma. Ann Cardiothorac Surg 2014;3:153-9.

14. Argueta AJ, Cañas SR, Zhu Y, et al. Bilateral uniportal VATS S3 segmentectomy. Asvide 2017;4:499. Available online: http://www.asvide.com/articles/1818 